\title{
Supplemental chromium-loaded chitosan nanoparticles affect growth, serum metabolites and intestinal histology in broilers
}

\author{
S.K. Tahir ${ }^{1}$, M.S. Yousaf ${ }^{1 \#}$, M.A. Rashid ${ }^{2}$, A.F. $\mathrm{Khan}^{3}$, S. Ahmad ${ }^{1}$, H. Zaneb ${ }^{4}$, I. $\mathrm{Khan}^{4}$ \& H. \\ Rehman $^{1}$ \\ ${ }^{1}$ Department of Physiology, University of Veterinary and Animal Sciences, Lahore, Pakistan \\ ${ }^{2}$ Department of Animal Nutrition, University of Veterinary and Animal Sciences, Lahore, Pakistan \\ ${ }^{3}$ Interdisciplinary Research Centre in Biomedical Materials, COMSATS Islamabad (Lahore Campus), Pakistan \\ ${ }^{4}$ Department of Anatomy and Histology, University of Veterinary and Animal Sciences, Lahore, Pakistan
}

(Received 15 July 2019; Accepted 5 January 2020; First published online 19 January 2020)

\author{
Copyright resides with the authors in terms of the Creative Commons Attribution 4.0 South African Licence. \\ See: http://creativecommons.org/licenses/by/4.0/za \\ Condition of use: The user may copy, distribute, transmit and adapt the work, but must recognise the authors and the South African \\ Journal of Animal Science.
}

\begin{abstract}
The goal of the present research was to evaluate the effects of chromium-loaded chitosan nanoparticles (Cr-CNPs) on production performance, viscera development, serum metabolites and intestinal histology in broilers. Two hundred (200) day-old broilers were randomly divided into five groups with five replicates $(n=8)$. Birds in the first group served as control and were fed a corn soybean-based diet, while the remaining four supplemented groups were offered 200, 400, 800, and $1200 \mu \mathrm{g} \mathrm{Cr}-\mathrm{CNPs} / \mathrm{kg}$ of feed, respectively, for 35 days. Weight gain, feed intake and feed conversion ratio (FCR) remained unaffected with $\mathrm{Cr}$-CNP supplementation. No changes were observed in the relative weights of viscera. The relative length of the small intestine was decreased in birds supplemented with 200 and $800 \mu \mathrm{g} \mathrm{Cr}-\mathrm{CNPs} / \mathrm{kg}$ compared with the $1200 \mu \mathrm{g} \mathrm{Cr}$-CNP-supplemented group and control. Serum metabolites remained unaffected with $\mathrm{Cr}$-CNP supplementation except for serum HDL, which was increased. Cr-CNPs decreased the retention of chromium in the bone at higher concentrations. Jejunal villus height, villus surface area, and villus height to crypt depth ratio were increased in the $800 \mu \mathrm{g}$ Cr-CNP-supplemented group. In conclusion, Cr-CNPs did not affect growth performance, viscera development, and most of the serum metabolites, but enhanced jejunal morphological attributes at $800 \mu \mathrm{g} \mathrm{Cr}-\mathrm{CNPs} / \mathrm{kg}$ of feed.
\end{abstract}

Keywords: blood biochemistry, health, nano-biotechnology, prebiotics, poultry, trace mineral

\#Corresponding author: drmshahbaz@uvas.edu.pk

\section{Introduction}

Chromium ( $\mathrm{Cr}$ ), though regarded as an important trace element and a constituent of the glucose tolerance factor is necessary for carbohydrate, fat, and protein metabolism (Mertz, 1993). Chromium supplementation is used to enhance growth performance, reproduction, carcass characteristics and tissue deposition in pigs and broilers (Page et al., 1993; Lindemann et al., 1995; Mooney \& Cromwell, 1995; Ghanbari et al., 2012). Most of the Cr contents of a corn-soybean diet are unavailable to animals owing to feed processing. The size of the particle, the nature of its polymers and zeta potential are some of the factors that determine the absorption rate through the intestine (Wang et al., 2007).

Chitosan is a non-toxic and biodegradable carbohydrate polymer and is well digested by birds (Hirano et al., 1990). Functional attributes of intestinal mucosa, including absorptive surface area, expression of brush border enzymes, and nutrient transport systems, are dependent on the shape and size of villi. Chitosan supplementation in birds showed improved intestinal morphology and increased villus size (Khambualai et al., 2009). Its beneficial effects on weight gain, FCR, and nitrogen retention were also investigated in broiler chickens and ducks (Shi et al., 2005; Shi-bin \& Hong, 2012).

The application of nanoparticles gained more attention because of their novel properties. Nanoparticles are different in properties from bulk materials because of small size, greater surface area, and shape of particles (Awad et al., 2012). Nano-composites have higher absorption rates in the gastrointestinal tract and are absorbed by gastrointestinal lymphatics (Desai et al., 1996; Hussain et al., 2001). Chromium (III) loaded chitosan nanoparticles have been reported to increase carcass lean percentage, decrease the fat 
percentage, and reduce backfat thickness in pigs (Wang \& Xu, 2004). They reduce the cholesterol levels in serum as they are involved in fatty acid metabolism and potentiate cellular, humoral, and mucosal immune responses (He et al., 2000). Positive effects of $\mathrm{Cr}$ nanoparticles on serum biochemistry, hormones, and immune status are also reported in pigs (Wang et al., 2007).

Organic salts of chromium have greater bioavailability than inorganic salts, but they are not cost effective. Also, prebiotics such as chitosan polysaccharides have been used extensively to improve the gut microbiota and morphology, under both normal and heat stress conditions. To minimize their dosage and excretion and enhance their absorption and solubility, the present research is planned to evaluate the effects of the supplementation of Cr-CNPs on growth performance, viscera development, serum metabolites and intestinal histology in broilers under standard conditions. To the best of the authors' knowledge, no research has been done on the use of Cr-CNPs in broilers, and only limited studies are available on pigs.

\section{Materials and Methods}

All the procedures adopted to perform this experiment were approved by the Ethical Review Committee of the University of Veterinary and Animal Sciences, Lahore, Pakistan, vide letter no. DR/498, dated 09-05-2018.

The Cr-CNPs were prepared and characterized at the Interdisciplinary Research Centre in Biomedical Research at COMSATS University, Islamabad (Lahore Campus), Pakistan, according to the method described by Wang et al. (2012). Briefly, $1 \%$ chitosan solution was prepared by dissolving chitosan in $0.5 \%$ acetic acid with $\mathrm{pH}$ adjusted at 3.5. Afterwards, the chitosan solution was stirred continuously for one hour and $200 \mathrm{mg} / \mathrm{L}$ chromium chloride solution was added to the chitosan solution during stirring to obtain a suspension of chitosan and chromium chloride. The $\mathrm{pH}$ of the suspension was adjusted to 6.5 and stirring continued for five hours. Then the precipitate was centrifuged at $12000 \mathrm{~g}$ for 15 minutes at room temperature and washed with water and dried to obtain Cr-CNPs.

Day-old broiler chicks $(n=200)$, purchased from a commercial hatchery, were randomly divided into five groups ( $n=40 /$ group), each group having five replicates $(n=8 /$ replicate). Birds in the first group were labelled the control group and offered a corn-soybean-based diet. Birds in the remaining four groups were labelled $200 \mathrm{Cr}$-CNPs, $400 \mathrm{Cr}-\mathrm{CNPs}, 800 \mathrm{Cr}$-CNPs, and $1200 \mathrm{Cr}$-CNPs, and were offered the same diet supplemented with graded levels of Cr-CNPs at the dose level of $200,400,800$ and $1200 \mu \mathrm{g} / \mathrm{kg}$ of feed, respectively, for 35 days. The feed and water were provided ad libitum. Temperature and relative humidity on day 1 were kept at $35 \pm 1.1{ }^{\circ} \mathrm{C}$ and $65 \pm 5 \%$, respectively. The temperature was decreased by $3{ }^{\circ} \mathrm{C}$ each week till it reached $26^{\circ} \mathrm{C}$. Birds were vaccinated against Newcastle disease and Infectious Bursal disease, as mentioned by Giambron and Clay (1986). The composition of the diet is presented in Table 1 (Khan et al., 2016).

Feed intake was measured daily, while bodyweight gain and FCR were determined weekly. On day 35 two birds from each replicate were randomly selected and slaughtered. A blood sample was collected and allowed to clot to collect serum that was stored at $-40^{\circ} \mathrm{C}$ until the analyses of serum metabolites. Viscera were collected to calculate the weights and lengths of the relative organs. The liver and whole tibial bone were stored at $-40^{\circ} \mathrm{C}$ to estimate their chromium contents. Intestinal segments (duodenum, jejunum, and ileum) were removed, washed with saline and stored in neutral buffered formalin for histology.

Prior to analysis, serum samples were thawed, vortexed and then analysed for lipid profile (total cholesterol, triglycerides and HDL-cholesterol), serum proteins (total proteins, albumin and globulins), urea and uric acid using commercial kits (HUMAN Gesellschaft für Biochemica und Diagnostica mbH, Wiesbaden, Germany) according to the manufacturer's recommendations using an Epoch $^{\text {TM }}$ micro-plate spectrophotometer (Biotek Instruments Inc., Winooski, USA). The serum alanine aminotransferase (ALT), aspartate aminotransferase (AST) and creatinine levels were determined by commercial kinetic kits (HUMAN Gesellschaft für Biochemica und Diagnostica $\mathrm{mbH}$, Wiesbaden, Germany, and Diasys, Holzheim, Germany) using UV/VIS spectrophotometer (UV-2800, Thermo Fischer Scientific, Waltham, Massachusetts, USA). Chromium concentration in samples was estimated using flame atomic absorption spectrophotometer (iCE 3300 double beam AA spectrometer, Thermo Fischer Scientific, Waltham, Massachusetts, USA) after wet digestion (Yousaf et al., 2009).

The intestinal mucosal morphometry was determined by analysing the duodenum, jejunum and ileum villus height, crypt depth, villus surface area, and villus height to crypt depth ratio. Intestines, collected from birds (two birds from each group), were processed according to a conventional method of haematoxylin and eosin (Ashraf et al., 2013) and examined using a light microscope (Olympus CX31, Olympus, Center Valley, Pennsylvania, USA) fitted with a digital imaging system (Olympus DP20, Olympus USA). Five villi with intact lamina propria and well orientation were used for observations. Villus height was measured from the tip of the villus to the villus crypt junction, and the crypt depth was measured from its base up to the region of 
transition between the crypt and villus. The villus surface area was measured by using the formula ( $2 p)$ (villus width/2)(villus length).

Table 1 Composition of the diet to be supplemented with chromium-loaded chitosan nanoparticles and fed to broilers

\begin{tabular}{lc}
\hline Ingredients (g/kg) & Percentage \\
\hline Corn & 58.50 \\
Soybean meal 44\% & 25.00 \\
Sunflower meal & 3.50 \\
Canola meal & 8.00 \\
Vegetable oil & 1.50 \\
Dicalcium phosphate & 0.90 \\
Limestone & 1.51 \\
Common salt & 0.50 \\
DL-Methionine & 0.21 \\
L-Lysine HCl & 0.12 \\
Vitamin premix & 0.13 \\
Micro mineral premix & 0.13 \\
Nutrient contents & \\
Crude protein & 20.72 \\
Metabolizable energy (MJ) & 12.20 \\
Calcium & 0.91 \\
Phosphorus & 0.61 \\
\hline
\end{tabular}

\footnotetext{
${ }^{1}$ Vitamins A (retinol): $11000 \mathrm{IU}$; $\mathrm{B}_{12}$ (cyanocobalamin): $0.0132 \mathrm{mg}$; $\mathrm{D}_{3}$ (cholecalciferol): $2200 \mathrm{IU}$; E (alpha-tocopherol): 22 IU; choline chloride: $440 \mathrm{mg}$; riboflavin: $8.8 \mathrm{mg}$; pantothenic acid: $22 \mathrm{mg}$; ethoxyquin: $250 \mathrm{mg}$; menadione: $2.2 \mathrm{mg}$; pyridoxine: $4.4 \mathrm{mg}$; folic acid: $1.1 \mathrm{mg}$; biotin" 0.22; thiamine: $4.4 \mathrm{mg}$

${ }^{2}$ Copper $\left(\mathrm{CuSO}_{4}\right): 20 \mathrm{mg}$; zinc (ZnO): $200 \mathrm{mg}$; manganese $\left(\mathrm{MnSO}_{4}\right): 240 \mathrm{mg}$; iron $\left(\mathrm{FeSO}_{4}\right): 120 \mathrm{mg}$; iodine (KI): 0.92 mg; calcium: $150-180 \mathrm{mg}$
}

Data were analysed statistically using SPSS for Windows version 20.0 (IBM Inc., Armonk, New York, USA). Data were presented as mean \pm SEM and analysed using one-way analysis of variance (ANOVA). For group differences, Duncan's multiple range test was used. Orthogonal contrasts were used to determine the linear, quadratic and cubic effects of $\mathrm{Cr}$-CNPs at $P<0.05$.

\section{Results}

The weight gain of birds supplemented with $200 \mu \mathrm{g} \mathrm{Cr-CNPs/kg}$ was found to be higher $(P<0.05)$ compared with the 800 , and $1200 \mathrm{Cr}$-CNPs and control groups during week 2 . But in weeks 1, 3, 4, and 5, no effect was found on weight gain in all the groups (Table 2). The mean feed intake remained unaffected by Cr-CNP-supplementation during all the weeks (Table 3). The FCR was significantly lower in birds supplemented with 400 and $200 \mu \mathrm{g} \mathrm{Cr}-\mathrm{CNPs} / \mathrm{kg}$ compared with the control and $1200 \mu \mathrm{g} \mathrm{Cr}-\mathrm{CNPs} / \mathrm{kg}$ supplemented groups during weeks 1 and 2, respectively. However, the FCR remained unchanged with $\mathrm{Cr}$ CNP supplementation in weeks 3,4 , and 5 as shown in Table 4. 
Table 2 Effects of supplementation with chromium-loaded chitosan nanoparticle on weekly weight gain $(\mathrm{g})$ in broilers $^{1}$

\begin{tabular}{|c|c|c|c|c|c|c|c|c|c|c|}
\hline \multirow[b]{2}{*}{ Weeks } & \multicolumn{5}{|c|}{ Treatments $^{2}$} & \multirow[b]{2}{*}{ SEM } & \multirow{2}{*}{$\begin{array}{c}P- \\
\text { Value }\end{array}$} & \multirow[b]{2}{*}{ Linear } & \multirow[b]{2}{*}{ Quadratic } & \multirow[b]{2}{*}{ Cubic } \\
\hline & Control & $\begin{array}{c}200 \mathrm{Cr}- \\
\mathrm{CNPS}\end{array}$ & $\begin{array}{l}400 \mathrm{Cr}- \\
\mathrm{CNPS}\end{array}$ & $\begin{array}{c}800 \mathrm{Cr}- \\
\mathrm{CNPS}\end{array}$ & $\begin{array}{c}1200 \mathrm{Cr}- \\
\mathrm{CNPS}\end{array}$ & & & & & \\
\hline Week 1 & 78 & 91 & 93 & 87 & 85 & 1.8 & 0.05 & 0.33 & 0.01 & 0.24 \\
\hline Week 2 & $184^{\mathrm{bc}}$ & $220^{a}$ & $203^{\mathrm{ab}}$ & $184^{b c}$ & $166^{\mathrm{C}}$ & 5.4 & 0.004 & 0.02 & 0.003 & 0.06 \\
\hline Week 3 & 345 & 353 & 359 & 350 & 317 & 8.2 & 0.57 & 0.33 & 0.19 & 0.73 \\
\hline Week 4 & 528 & 576 & 544 & 531 & 534 & 12.8 & 0.80 & 0.75 & 0.55 & 0.34 \\
\hline Week 5 & 625 & 594 & 481 & 571 & 482 & 23.8 & 0.17 & 0.07 & 0.63 & 0.56 \\
\hline
\end{tabular}

Table 3 Effects of supplementation with chromium-loaded chitosan nanoparticles on weekly feed intake (g) in broilers ${ }^{1}$

\begin{tabular}{|c|c|c|c|c|c|c|c|c|c|c|}
\hline \multirow[b]{2}{*}{ Weeks } & \multicolumn{5}{|c|}{ Treatments $^{2}$} & \multirow[b]{2}{*}{ SEM } & \multirow[b]{2}{*}{$P$-Value } & \multirow[b]{2}{*}{ Linear } & \multirow[b]{2}{*}{ Quadratic } & \multirow[b]{2}{*}{ Cubic } \\
\hline & Control & $\begin{array}{l}200 \mathrm{Cr}- \\
\mathrm{CNPS}\end{array}$ & $\begin{array}{l}400 \mathrm{Cr}- \\
\mathrm{CNPS}\end{array}$ & $\begin{array}{l}800 \mathrm{Cr}- \\
\mathrm{CNPS}\end{array}$ & $\begin{array}{l}1200 \mathrm{Cr}- \\
\mathrm{CNPS}\end{array}$ & & & & & \\
\hline Week 1 & 126 & 138 & 137 & 135 & 137 & 1.7 & 0.13 & 0.10 & 0.12 & 0.13 \\
\hline Week 2 & 311 & 323 & 320 & 284 & 291 & 5.6 & 0.07 & 0.03 & 0.30 & 0.11 \\
\hline Week 3 & 631 & 613 & 629 & 599 & 542 & 14.4 & 0.29 & 0.07 & 0.31 & 0.54 \\
\hline Week 4 & 870 & 912 & 862 & 917 & 819 & 15.1 & 0.29 & 0.36 & 0.16 & 0.56 \\
\hline Week 5 & 1244 & 1196 & 1075 & 1182 & 1007 & 35.3 & 0.17 & 0.05 & 0.92 & 0.39 \\
\hline
\end{tabular}

${ }^{1}$ Data presented as mean \pm SEM of five replicated $(n=8$ birds/replicate)

${ }^{2}$ Control: without chromium loaded chitosan nanoparticles (Cr-CNPs); $200 \mathrm{Cr}$-CNPs: fed with $200 \mu \mathrm{g}$ Cr-CNPs per kg of feed; 400 Cr-CNPs: fed with $400 \mu \mathrm{g} \mathrm{Cr}$-CNPs per kg of feed; $800 \mathrm{Cr}$-CNPs: fed with $800 \mu \mathrm{C} \mathrm{Cr-CNPs} \mathrm{per} \mathrm{kg} \mathrm{of} \mathrm{feed;}$ 1200 Cr-CNPs: fed with $1200 \mu \mathrm{g}$ Cr-CNPs per kg of feed

Table 4 Effects of supplementation with chromium-loaded chitosan nanoparticles on weekly feed conversion ratio in broilers ${ }^{1}$

\begin{tabular}{|c|c|c|c|c|c|c|c|c|c|c|}
\hline \multirow[b]{2}{*}{ Weeks } & \multicolumn{5}{|c|}{ Treatments $^{2}$} & \multirow[b]{2}{*}{ SEM } & \multirow{2}{*}{$\begin{array}{c}P- \\
\text { Value }\end{array}$} & \multirow[b]{2}{*}{ Linear } & \multirow[b]{2}{*}{ Quadratic } & \multirow[b]{2}{*}{ Cubic } \\
\hline & Control & $\begin{array}{l}200 \mathrm{Cr}- \\
\mathrm{CNPS}\end{array}$ & $\begin{array}{l}400 \mathrm{Cr}- \\
\mathrm{CNPS}\end{array}$ & $\begin{array}{l}800 \mathrm{Cr}- \\
\mathrm{CNPS}\end{array}$ & $\begin{array}{l}1200 \mathrm{Cr}- \\
\text { CNPs }\end{array}$ & & & & & \\
\hline Week 1 & $1.63^{\mathrm{a}}$ & $1.53^{\mathrm{ab}}$ & $1.48^{b}$ & $1.55^{\mathrm{ab}}$ & $1.62^{\mathrm{a}}$ & 0.02 & 0.03 & 0.92 & 0.002 & 0.67 \\
\hline Week 2 & $1.69^{\mathrm{ab}}$ & $1.47^{c}$ & $1.58^{\mathrm{bc}}$ & $1.55^{\mathrm{bc}}$ & $1.76^{\mathrm{a}}$ & 0.03 & 0.01 & 0.22 & 0.002 & 0.55 \\
\hline Week 3 & 1.84 & 1.73 & 1.78 & 1.72 & 1.71 & 0.03 & 0.70 & 0.26 & 0.69 & 0.64 \\
\hline Week 4 & 1.67 & 1.60 & 1.60 & 1.73 & 1.54 & 0.04 & 0.57 & 0.59 & 0.73 & 0.17 \\
\hline Week 5 & 2.00 & 2.01 & 2.26 & 2.08 & 2.12 & 0.04 & 0.34 & 0.33 & 0.33 & 0.94 \\
\hline
\end{tabular}

\footnotetext{
${ }^{1}$ Data presented as mean \pm SEM of five replicated $(n=8$ birds/replicate)

${ }^{\mathrm{a}-\mathrm{c}}$ Within the row different superscript indicates significantly different means at $P<0.05$

${ }^{2}$ Control: Without chromium-loaded chitosan nanoparticles (Cr-CNPs); $200 \mathrm{Cr}-\mathrm{CNPs}$ : fed with $200 \mu \mathrm{Cg}$-CNPs per kg of feed; $400 \mathrm{Cr}$-CNPs: fed with $400 \mu \mathrm{g} \mathrm{Cr}$-CNPs per kg of feed; $800 \mathrm{Cr}$-CNPs: fed with $800 \mu \mathrm{g}$ Cr-CNPs per kg of feed; 1200 Cr-CNPs: fed with $1200 \mu \mathrm{g}$ Cr-CNPs per kg of feed
} 
The relative viscera weights and caecal length remained unaffected with Cr-CNP supplementation. But, the relative length of the small intestine was significantly reduced in birds supplemented with 200 and $800 \mu \mathrm{g} \mathrm{Cr}-\mathrm{CNPs} / \mathrm{kg}$ compared with the $1200 \mu \mathrm{g} \mathrm{Cr-CNPs/kg}$ supplemented and control groups (Table 5). The serum metabolites remained unaffected with Cr-CNP supplementation except for the serum HDLcholesterol which was increased $(P<0.05)$ with $\mathrm{Cr}$-CNP supplementation compared with the control group (Table 6). The chromium concentration in serum and liver remained unchanged with $\mathrm{Cr}-\mathrm{CNP}$ supplementation while in bone, the $\mathrm{Cr}$ concentration was decreased $(P<0.001)$ in $1200 \mu \mathrm{g} \mathrm{Cr}-\mathrm{CNPs} / \mathrm{kg}$ compared with the $200 \mu \mathrm{g} \mathrm{Cr}$-CNPs/kg supplemented and control groups (Table 7).

Table 5 Effects of supplementation with chromium-loaded chitosan nanoparticles on relative viscera weights and lengths in broilers

\begin{tabular}{|c|c|c|c|c|c|c|c|c|c|c|}
\hline & \multicolumn{5}{|c|}{ Treatments $^{2}$} & \multirow[b]{2}{*}{ SEM } & \multirow[b]{2}{*}{$\begin{array}{c}P- \\
\text { Value }\end{array}$} & \multirow[b]{2}{*}{ Linear } & \multirow[b]{2}{*}{ Quadratic } & \multirow[b]{2}{*}{ Cubic } \\
\hline & Control & $\begin{array}{l}200 \mathrm{Cr}- \\
\text { CNPs }\end{array}$ & $\begin{array}{l}400 \mathrm{Cr}- \\
\text { CNPs }\end{array}$ & $\begin{array}{l}800 \mathrm{Cr}- \\
\text { CNPs }\end{array}$ & $\begin{array}{c}1200 \\
\text { Cr- } \\
\text { CNPS }\end{array}$ & & & & & \\
\hline \multicolumn{11}{|c|}{ Organs' weight ${ }^{3}$} \\
\hline Liver & 3.21 & 2.66 & 2.54 & 2.59 & 2.48 & 0.10 & 0.12 & 0.03 & 0.11 & 0.38 \\
\hline Pancreas & 0.27 & 0.26 & 0.24 & 0.25 & 0.26 & 0.01 & 0.31 & 0.60 & 0.12 & 0.56 \\
\hline Gizzard & 1.88 & 1.85 & 1.71 & 1.90 & 2.01 & 0.04 & 0.13 & 0.21 & 0.05 & 0.92 \\
\hline Proventriculus & 0.44 & 0.45 & 0.37 & 0.43 & 0.44 & 0.01 & 0.19 & 0.78 & 0.18 & 0.69 \\
\hline Heart & 0.48 & 0.98 & 0.43 & 0.47 & 0.44 & 0.10 & 0.36 & 0.42 & 0.57 & 0.17 \\
\hline Spleen & 0.20 & 0.13 & 0.10 & 0.13 & 0.15 & 0.02 & 0.49 & 0.48 & 0.11 & 0.62 \\
\hline Bursa & 0.05 & 0.05 & 0.05 & 0.05 & 0.06 & 0.01 & 0.68 & 0.29 & 0.61 & 0.38 \\
\hline Caecal Tonsils & 0.02 & 0.02 & 0.02 & 0.01 & 0.02 & 0.01 & 0.73 & 0.57 & 0.58 & 0.41 \\
\hline Small Intestine & 2.26 & 2.21 & 2.19 & 2.48 & 2.37 & 0.08 & 0.78 & 0.40 & 0.81 & 0.46 \\
\hline Caecum & 0.13 & 0.13 & 0.14 & 0.12 & 0.13 & 0.01 & 0.95 & 0.98 & 0.96 & 0.72 \\
\hline \multicolumn{11}{|c|}{ Intestinal Length ${ }^{4}$} \\
\hline Small Intestine & $9.95^{\mathrm{a}}$ & $8.20^{\mathrm{b}}$ & $9.16^{\mathrm{ab}}$ & $8.36^{\mathrm{b}}$ & $9.95^{\mathrm{a}}$ & 0.23 & 0.02 & 0.92 & 0.01 & 0.83 \\
\hline Caecum & 0.99 & 0.82 & 0.87 & 0.87 & 0.93 & 0.02 & 0.23 & 0.72 & 0.06 & 0.30 \\
\hline
\end{tabular}

${ }^{1}$ Data were presented as mean \pm SEM of five replicated $(n=8$ birds/replicate)

${ }^{a-b}$ Within the row different superscript indicates significantly different means at $P<0.05$

${ }^{2}$ Control: without chromium-loaded chitosan nanoparticles (Cr-CNPs); $200 \mathrm{Cr}$-CNPs: fed with $200 \mu \mathrm{g}$ Cr-CNPs per kg of feed; 400 Cr-CNPs: fed with $400 \mu \mathrm{g} \mathrm{Cr-CNPs} \mathrm{per} \mathrm{kg} \mathrm{of} \mathrm{feed;} 800 \mathrm{Cr}$-CNPs: fed with $800 \mu \mathrm{g}$ Cr-CNPs per kg of feed; 1200 Cr-CNPs: fed with $1200 \mu \mathrm{g}$ Cr-CNPs per kg of feed

${ }^{3}$ Relative weight $=(\text { organ weight } / \text { body weight })^{\star} 100$

${ }^{4}$ Relative length $=$ (organ length/body weight ${ }^{\star} 100$ 
Table 6 Effects of supplementation with chromium-loaded chitosan nanoparticles on serum biochemical metabolites in broilers ${ }^{1}$

\begin{tabular}{|c|c|c|c|c|c|c|c|c|c|c|}
\hline \multirow[b]{2}{*}{ Parameters } & \multicolumn{5}{|c|}{ Treatments $^{2}$} & \multirow[b]{2}{*}{ SEM } & \multirow{2}{*}{$\begin{array}{c}P- \\
\text { Value }\end{array}$} & \multirow[b]{2}{*}{ Linear } & \multirow[b]{2}{*}{ Quadratic } & \multirow[b]{2}{*}{ Cubic } \\
\hline & Control & $\begin{array}{l}200 \mathrm{Cr}- \\
\text { CNPs }\end{array}$ & $\begin{array}{l}400 \mathrm{Cr}- \\
\mathrm{CNPs}\end{array}$ & $\begin{array}{l}800 \mathrm{Cr}- \\
\mathrm{CNPs}\end{array}$ & $\begin{array}{c}1200 \mathrm{Cr}- \\
\mathrm{CNPs}\end{array}$ & & & & & \\
\hline $\begin{array}{l}\text { Cholesterol } \\
\text { (mg/dL) }\end{array}$ & 126.52 & 141.48 & 131.56 & 143.11 & 147.70 & 2.89 & 0.09 & 0.03 & 0.97 & 0.38 \\
\hline $\begin{array}{l}\text { Triglycerides } \\
(\mathrm{mg} / \mathrm{dL})\end{array}$ & 195.49 & 201.74 & 202.92 & 203.65 & 211.11 & 2.31 & 0.28 & 0.04 & 0.92 & 0.48 \\
\hline $\mathrm{HDL}(\mathrm{mg} / \mathrm{dL})$ & $36.07^{b}$ & $56.6^{\mathrm{a}}$ & $46.69^{a}$ & $49.22^{\mathrm{a}}$ & $53.63^{a}$ & 2.22 & 0.02 & 0.04 & 0.21 & 0.03 \\
\hline $\operatorname{ALT}(\mathrm{U} / \mathrm{L})$ & 15.44 & 13.41 & 15.44 & 15.89 & 11.30 & 0.74 & 0.22 & 0.25 & 0.27 & 0.09 \\
\hline AST (U/L) & 50.11 & 61.26 & 61.95 & 56.52 & 62.00 & 1.81 & 0.15 & 0.11 & 0.23 & 0.08 \\
\hline $\begin{array}{l}\text { Total Proteins } \\
(\mathrm{g} / \mathrm{dL})\end{array}$ & 4.72 & 4.90 & 5.06 & 4.79 & 4.96 & 0.06 & 0.44 & 0.41 & 0.37 & 0.38 \\
\hline Albumin (g/dL) & 3.08 & 2.97 & 3.02 & 3.24 & 3.07 & 0.03 & 0.32 & 0.33 & 0.82 & 0.06 \\
\hline $\begin{array}{l}\text { Globulins } \\
\text { (g/dL) }\end{array}$ & 1.64 & 1.92 & 2.05 & 1.56 & 1.89 & 0.07 & 0.25 & 0.80 & 0.38 & 0.10 \\
\hline A/G Ratio & 2.03 & 1.56 & 1.52 & 2.31 & 1.65 & 0.10 & 0.09 & 0.97 & 0.54 & 0.02 \\
\hline $\begin{array}{l}\text { Creatinine } \\
\text { (mg/dL) }\end{array}$ & 1.70 & 1.76 & 1.74 & 2.03 & 2.05 & 0.22 & 0.98 & 0.55 & 0.91 & 0.90 \\
\hline Urea (mg/dL) & 29.39 & 27.50 & 29.44 & 29.67 & 28.33 & 0.76 & 0.90 & 0.99 & 0.93 & 0.41 \\
\hline $\begin{array}{l}\text { Uric Acid } \\
\text { (mg/dL) }\end{array}$ & 6.23 & 6.37 & 6.25 & 6.65 & 5.61 & 0.20 & 0.25 & 0.33 & 0.10 & 0.31 \\
\hline
\end{tabular}

${ }^{1}$ Data presented as mean \pm SEM of five replicated ( $n=8$ birds/replicate)

${ }^{\mathrm{a}-\mathrm{b}}$ Within the row different superscript indicates significantly different means at $P<0.05$

${ }^{2}$ Control: without chromium-loaded chitosan nanoparticles (Cr-CNPs); $200 \mathrm{Cr}$-CNPs: fed with $200 \mu \mathrm{g}$ Cr-CNPs per kg of feed; $400 \mathrm{Cr}$-CNPs: fed with $400 \mu \mathrm{g} \mathrm{Cr-CNPs}$ per kg of feed; $800 \mathrm{Cr}$-CNPs: fed with $800 \mu \mathrm{g}$ Cr-CNPs per kg of feed; 1200 Cr-CNPs: fed with $1200 \mu \mathrm{g}$ Cr-CNPs per kg of feed

ALT: serum alanine aminotransferase, AST: aspartate aminotransferase

Table 7 Effects of supplementation with chromium-loaded chitosan nanoparticles on chromium concentration $(\mathrm{ppm})$ in various analytes in broilers ${ }^{1}$

\begin{tabular}{|c|c|c|c|c|c|c|c|c|c|c|}
\hline \multirow[b]{2}{*}{ Analytes } & \multicolumn{5}{|c|}{ Treatments $^{2}$} & \multirow[b]{2}{*}{ SEM } & \multirow{2}{*}{$\begin{array}{c}P- \\
\text { Value }\end{array}$} & \multirow[b]{2}{*}{ Linear } & \multirow[b]{2}{*}{ Quadratic } & \multirow[b]{2}{*}{ Cubic } \\
\hline & Control & $\begin{array}{l}200 \mathrm{Cr}- \\
\text { CNPs }\end{array}$ & $\begin{array}{l}400 \mathrm{Cr}- \\
\mathrm{CNPs}\end{array}$ & $\begin{array}{c}800 \mathrm{Cr}- \\
\mathrm{CNPs}\end{array}$ & $\begin{array}{c}1200 \mathrm{Cr}- \\
\text { CNPs }\end{array}$ & & & & & \\
\hline Serum & 17.68 & 14.54 & 13.91 & 17.41 & 11.99 & 0.92 & 0.32 & 0.24 & 0.96 & 0.13 \\
\hline Liver & 14.97 & 19.06 & 14.48 & 18.57 & 18.97 & 0.89 & 0.26 & 0.23 & 0.86 & 0.46 \\
\hline Bone & $11.49^{b}$ & $23.98^{\mathrm{a}}$ & $7.16^{\mathrm{bc}}$ & $8.95^{\mathrm{bc}}$ & $2.47^{\mathrm{c}}$ & 1.89 & $<0.001$ & 0.002 & 0.07 & 0.01 \\
\hline
\end{tabular}

${ }^{1}$ Data presented as mean \pm SEM of five replicated $(n=8$ birds/replicate)

${ }^{\mathrm{a}-\mathrm{c}}$ Within the row different superscript indicates significantly different means at $P<0.05$

${ }^{2}$ Control: without Cr-CNPs; 200 Cr-CNPs: fed with $200 \mu \mathrm{g} \mathrm{Cr-CNPs} \mathrm{per} \mathrm{kg} \mathrm{of} \mathrm{feed;} 400 \mathrm{Cr}-\mathrm{CNPs}$ : fed with $400 \mu \mathrm{g}$ CrCNPs per kg of feed; 800 Cr-CNPs: fed with $800 \mu \mathrm{g}$ Cr-CNPs per kg of feed; 1200 Cr-CNPs: fed with $1200 \mu \mathrm{g}$ Cr-CNPs per $\mathrm{kg}$ of feed

The results of intestinal micro-architecture in various segments of the small intestine are shown in Table 8. Villus height of duodenum and jejunum was significantly increased with $800 \mu \mathrm{g} \mathrm{Cr}$-CNP supplementation compared with $1200 \mu \mathrm{g} \mathrm{Cr}-\mathrm{CNPs} / \mathrm{kg}$ supplemented group, while ileal villus height remained unchanged. Crypt depth of duodenum was increased $(P<0.001)$ with 200 and $400 \mu \mathrm{g} \mathrm{Cr}$-CNP supplementation, but no effects were observed in jejunal and ileal crypt depth with Cr-CNP supplementation. The villus width, villus surface area, and villus height to crypt depth ratio remained unaffected in duodenum and ileum with $\mathrm{Cr}$-CNP supplementation. However, villus width and villus surface area in the jejunum of birds supplemented with 400 and $800 \mu \mathrm{g} \mathrm{Cr-CNPs/kg} \mathrm{was} \mathrm{found} \mathrm{higher}(P<0.001)$ compared with the control 
group. Villus height to crypt depth ratio of jejunum in birds supplemented with $800 \mu \mathrm{g} \mathrm{Cr}$-CNPs/kg was found higher $(P<0.05)$ compared with the 1200 Cr-CNPs group.

Table 8 Effects of supplementation with chromium-loaded chitosan nanoparticles on intestinal microarchitecture in broilers ${ }^{1}$

\begin{tabular}{|c|c|c|c|c|c|c|c|c|c|c|}
\hline \multirow{2}{*}{ Parameters } & \multicolumn{5}{|c|}{ Treatments $^{2}$} & \multirow{2}{*}{ SEM } & \multirow{2}{*}{$P$-Value } & \multirow{2}{*}{ Linear } & \multirow{2}{*}{ Quadratic } & \multirow[b]{2}{*}{ Cubic } \\
\hline & Control & $\begin{array}{l}200 \mathrm{Cr}- \\
\mathrm{CNPs}\end{array}$ & $\begin{array}{l}400 \mathrm{Cr}- \\
\mathrm{CNPs}\end{array}$ & $\begin{array}{l}800 \mathrm{Cr}- \\
\mathrm{CNPs}\end{array}$ & $\begin{array}{c}1200 \mathrm{Cr}- \\
\mathrm{CNPs}\end{array}$ & & & & & \\
\hline \multicolumn{11}{|c|}{ Duodenum } \\
\hline $\mathrm{VH}^{3}(\mu \mathrm{m})$ & $1230^{\mathrm{a}}$ & $899^{\mathrm{bc}}$ & $955^{\mathrm{ab}}$ & $1226^{\mathrm{a}}$ & $624^{c}$ & 66.01 & 0.001 & 0.01 & 0.39 & 0.002 \\
\hline $\mathrm{VW}(\mu \mathrm{m})$ & 77 & 68 & 64 & 49 & 69 & 3.30 & 0.06 & 0.11 & 0.09 & 0.17 \\
\hline $\mathrm{CD}(\mu \mathrm{m})$ & $118^{\mathrm{b}}$ & $227^{\mathrm{a}}$ & $248^{a}$ & $150^{b}$ & $144^{b}$ & 10.79 & $<0.001$ & 0.57 & $<0.001$ & 0.001 \\
\hline VSA $\left(\mathrm{mm}^{2}\right)$ & 0.24 & 0.17 & 0.20 & 0.16 & 0.17 & 0.01 & 0.18 & 0.06 & 0.38 & 0.56 \\
\hline VH:CD & 8.69 & 5.12 & 5.11 & 5.83 & 5.38 & 0.44 & 0.13 & 0.07 & 0.06 & 0.12 \\
\hline \multicolumn{11}{|c|}{ Jejunum } \\
\hline $\mathrm{VH}(\mu \mathrm{m})$ & $464^{b}$ & $513^{a b}$ & $635^{\mathrm{ab}}$ & $645^{a}$ & $473^{b}$ & 23.52 & 0.02 & 0.47 & 0.02 & 0.13 \\
\hline $\mathrm{VW}(\mu \mathrm{m})$ & $49^{c}$ & $48^{c}$ & $83^{a}$ & $65^{b}$ & $61^{\mathrm{bc}}$ & 2.42 & $<0.001$ & 0.02 & 0.003 & 0.12 \\
\hline $\mathrm{CD}(\mu \mathrm{m})$ & 100 & 130 & 119 & 109 & 124 & 4.99 & 0.35 & 0.57 & 0.54 & 0.08 \\
\hline VSA $\left(\mathrm{mm}^{2}\right)$ & $0.07^{\mathrm{b}}$ & $0.08^{\mathrm{b}}$ & $0.16^{\mathrm{a}}$ & $0.13^{\mathrm{a}}$ & $0.09^{b}$ & 0.01 & $<0.001$ & 0.11 & 0.001 & 0.04 \\
\hline $\mathrm{VH}: \mathrm{CD}$ & $4.81^{\mathrm{ab}}$ & $4.65^{\mathrm{ab}}$ & $5.67^{\mathrm{ab}}$ & $6.49^{\mathrm{a}}$ & $3.80^{\mathrm{b}}$ & 0.26 & 0.03 & 0.94 & 0.04 & 0.02 \\
\hline \multicolumn{11}{|c|}{ Ileum } \\
\hline $\mathrm{VH}(\mu \mathrm{m})$ & 535 & 447 & 536 & 502 & 451 & 12.88 & 0.39 & 0.23 & 0.65 & 0.49 \\
\hline $\mathrm{VW}(\mu \mathrm{m})$ & 78 & 72 & 79 & 75 & 82 & 2.14 & 0.66 & 0.49 & 0.49 & 0.94 \\
\hline $\mathrm{CD}(\mu \mathrm{m})$ & 147 & 132 & 156 & 130 & 146 & 3.90 & 0.19 & 0.90 & 0.70 & 0.93 \\
\hline VSA $\left(\mathrm{mm}^{2}\right)$ & 1.30 & 1.02 & 1.35 & 1.19 & 1.23 & 0.05 & 0.17 & 0.97 & 0.73 & 0.28 \\
\hline $\mathrm{VH}: \mathrm{CD}$ & 3.91 & 3.64 & 3.62 & 3.92 & 3.18 & 0.11 & 0.32 & 0.16 & 0.52 & 0.15 \\
\hline
\end{tabular}

${ }^{1}$ Data presented as mean \pm SEM of five replicated $(n=8$ birds/replicate)

${ }^{a-c}$ within the row different superscript indicates significantly different means at $P<0.05$

${ }^{2}$ Control: without chromium-loaded chitosan nanoparticles (Cr-CNPs); $200 \mathrm{Cr}$-CNPs: fed with $200 \mu \mathrm{Cg}$-CNPs per kg of feed; $400 \mathrm{Cr}$-CNPs: fed with $400 \mu \mathrm{g}$ Cr-CNPs per kg of feed; $800 \mathrm{Cr}$-CNPs: fed with $800 \mu \mathrm{g}$ Cr-CNPs per kg of feed; 1200 Cr-CNPs: fed with $1200 \mu \mathrm{g}$ Cr-CNPs per kg of feed

${ }^{3} \mathrm{VH}$ : villus height; VW: villus width; CD: crypt depth; VSA: villus surface area; VH:CD: villus height to crypt depth ratio

\section{Discussion}

Improved production performance with $\mathrm{Cr}$ supplementation is reported in some experiments in broilers, steers and pigs (Rosebrough \& Steele, 1981; Chang et al., 1992; Page et al., 1993; Lien et al.,1999), while others reported no effect (Kegly et al., 1996). In the current study, no change was observed in weight gain, feed intake and FCR with Cr-CNP supplementation in weeks $1,3,4$, and 5 . Unayik et al. (2002) reported no significant effect of $\mathrm{Cr}$ supplementation on the bodyweight and weight gain of broilers supplemented with inorganic chromium chloride, but they reported reduced feed intake and improved feed efficiency at the dose of $20 \mathrm{mg} / \mathrm{kg}$ chromium (III) chloride $\left(\mathrm{CrCl}_{3}\right)$. Sirirat et al. (2012) also found no effect of nano-chromium picolinate supplementation on the bodyweight and weight gain but observed decreased feed intake at 500 and $3000 \mathrm{ppb}$ nano-chromium picolinate and improved FCR at $3000 \mathrm{ppb}$ nano-chromium picolinate in broilers. Lin et al. (2015) also found no effect on bodyweight and FCR of broilers with $\mathrm{Cr}$ supplementation from either organic or inorganic sources. Zheng et al. (2016) also reported no effect of $\mathrm{Cr}$ supplementation from Cr-picolinate, Cr-propionate and $\mathrm{CrCl}_{3}$ at the concentration of 0.4 and $2 \mathrm{mg} / \mathrm{kg}$ feed on the weight gain, feed intake and FCR of broilers. Rajalekshmi et al. (2014) also reported no change in weight gain, feed intake, or FCR with various dietary concentrations of $\mathrm{Cr}$-picolinate ranging from 0 to $3.2 \mathrm{mg} \mathrm{Cr} / \mathrm{kg}$ diet for 42 days kept under normal conditions. Similarly, Wang et al. (2012) reported that Cr-CNPs (100, 200 and $400 \mu \mathrm{g} / \mathrm{kg}$ feed) supplementation did not affect the performance of the finishing pigs. Under heat stress 
conditions, $\mathrm{Cr}$ supplementation from organic and inorganic sources did not affect bodyweight, feed intake and FCR (Amatya et al., 2004; Moeini et al., 2011; Toghyani et al., 2012). The effect on the growth performance of broilers with $\mathrm{Cr}$ supplementation remained inconsistent (Rajalekshmi et al. 2014). However, the differences in studies in terms of growth performance could be because of the differences in the breeds or species, and source and size of chromium, which that greatly affect the bioavailability, as well as environmental variations.

The relative viscera weights and caecal length were not affected by $\mathrm{Cr}$-CNPs supplementation in this study. However, the relative length of the small intestine was decreased significantly in birds supplemented with 200 and $800 \mu \mathrm{g} \mathrm{Cr}-\mathrm{CNPs} / \mathrm{kg}$ of feed. The relative weight of liver and bursa was increased with $\mathrm{CrCl}_{3}$ supplementation (Unayik et al., 2002), but no effect was observed on the relative weight of liver and spleen in broilers with nano Cr-picolinate supplementation (Sirirat et al., 2012). Similarly, no effect was found in the liver and pancreas weights in chicks fed with Cr-picolinate (Ghanbari et al., 2012). However, the relative weight of liver, heart, spleen, and gizzards was increased linearly with supplementation of Cr-picolinate in broilers reared under heat stress (Sahin et al., 2002), but no effect was seen on the relative mass of liver (Moeini et al., 2011; Toghyani et al., 2012; Akbari \& Torki, 2014), bursa (Moeini et al., 2011), spleen (Moeini et al., 2011; Hamidi et al., 2016), pancreas (Toghyani et al., 2012), and heart (Moeini et al., 2011; Habibian et al., 2013; Hamidi et al., 2016) in birds reared during heat stress conditions.

Serum cholesterol and triglycerides were not affected by Cr-CNP supplementation, but serum HDLcholesterol was increased linearly and cubically in the present research. Similar observations were reported by Uyanik et al. (2002). They found no effect on serum cholesterol concentration with $\mathrm{CrCl}_{3}$ supplementation at the doses of 20,40 and $80 \mathrm{mg} / \mathrm{kg}$ of feed in broilers. Motozono et al. (1998) also found no effect on serum cholesterol and triglycerides levels with the addition of Cr-picolinate to the diet. Lin et al. (2015) reported no effect of trivalent chromium supplementation on serum cholesterol and HDL but decreased triglycerides concentration with $1200 \mu \mathrm{gg} / \mathrm{kg} \mathrm{CrCl}_{3}$. In another study by Wang et al. (2012) on Cr-CNP supplementation at the levels of 100,200 , and $400 \mu \mathrm{g} \mathrm{Cr}-\mathrm{CNP} / \mathrm{kg}$ in pigs reported no effect on serum cholesterol, triglycerides, and HDL levels, but Moeini et al. (2011) reported a slight improvement in the serum HDL with $\mathrm{Cr}$ supplementation in broilers. However, under heat stress, $\mathrm{Cr}$ supplementation reduces the serum cholesterol levels in broilers (Sahin et al., 2002; Habibian et al., 2013) and in quails (Sahin et al., 2005). These variations in results can be owing to the different sources of $\mathrm{Cr}$ being supplemented and therefore need further insights. The serum total proteins, albumin, globulin concentrations and the albumin to globulin ratio remained unaffected with Cr-CNP supplementation. Some studies also indicated no effect on serum proteins with $\mathrm{Cr}$ as Cr-yeast (Kroliczewska et al., 2004) and $\mathrm{CrCl}_{3}$ (Bakhiet et al., 2007; Samanta et al., 2008) in boilers and as Cr-CNP (Wang et al., 2012) in pigs, but other studies reported an increase in serum proteins with supplementation of $\mathrm{Cr}$ as $\mathrm{CrCl}_{3}$ (Unayik et al., 2002; Ahmed et al., 2005) in broilers. During heat stress, serum proteins were increased with $\mathrm{Cr}$ supplementation as Cr-picolinate (Sahin et al., 2002) but there was no effect on serum albumin (Akbari \& Torki, 2014). Serum urea, creatinine, and uric acid were assessed to investigate $\mathrm{Cr}$ toxicity, but $\mathrm{Cr}$ as $\mathrm{Cr}$-CNPs did not affect the urea, creatinine and uric acid, which indicates that supplemented doses of $\mathrm{Cr}$ were not nephrotoxic. Serum ALT and AST were not changed with Cr-CNP supplementation, which indicates the supplemented doses of Cr-CNPs were not hepatotoxic. Wang et al. (2012) also reported that serum ALT and AST remained unchanged with the supplementation of $\mathrm{Cr}$ as $\mathrm{Cr}$ CNP in pigs. The current results are in line with those of Bakhiet et al. (2007), who found no effect of $\mathrm{Cr}$ supplementation as $\mathrm{CrCl}_{3}$ on serum AST and uric acid in broilers.

In the present study, $\mathrm{Cr}$ concentration in serum and liver remained unchanged with $\mathrm{Cr}$ supplementation, but $\mathrm{Cr}$ retention was lower in the bone of birds supplemented with $1200 \mu \mathrm{g} \mathrm{Cr}$-CNPs. The current results are in line with the study conducted by Kroliczewska et al. (2004), who reported no effect on serum $\mathrm{Cr}$ in chicks supplemented with $\mathrm{Cr}$-yeast at the level of 300 and $500 \mu \mathrm{g} / \mathrm{kg}$ of diet. Similarly, Lin et al. (2015) observed no change in serum $\mathrm{Cr}$ of broilers supplemented with $\mathrm{CrCl}_{3}$ at the dose of $1200 \mu \mathrm{g} / \mathrm{kg}$, but broilers supplemented with $\mathrm{Cr}$ as $\mathrm{Cr}$-picolinate and nano-Cr-picolinate had increased serum chromium at the dose of $1200 \mu \mathrm{g} / \mathrm{kg}$ of diet. Toghyani et al. (2012) found that liver $\mathrm{Cr}$ remains unaffected in birds supplemented with $\mathrm{CrCl}_{3}$ at the levels of 500,1000 , or $1500 \mu \mathrm{g} / \mathrm{kg}$ diet or Cr-nickel at the levels of 500 or $1000 \mu \mathrm{g} / \mathrm{kg}$ diet, but increased with Cr-nickel at the level of $1500 \mu \mathrm{g} / \mathrm{kg}$ diet. Similarly, Zha et al. (2009) observed an increase in liver retention of $\mathrm{Cr}$ in birds supplemented with $\mathrm{CrCl}_{3}, \mathrm{Cr}$-picolinate or $\mathrm{Cr}$ nanoparticles at the level of $500 \mu \mathrm{g} / \mathrm{kg}$ diet. However, Amatya et al. (2004) found a decrease in liver $\mathrm{Cr}$ concentration with $\mathrm{Cr}$ supplementation as $\mathrm{CrCl}_{3}$ or Cr-yeast at the dose of $200 \mu \mathrm{g} / \mathrm{kg}$ diet. In another study by Wang et al. (2012), they reported that $\mathrm{Cr}$ retention in blood, muscle, liver, kidney, heart, and pancreas increased linearly with $\mathrm{Cr}$ supplementation as $\mathrm{Cr}$-CNP in pigs.

Improvement in the mucosal morphology of the gut is regarded as a health indicator and growth in birds (Awad et al., 2009). The intestine mucosal barrier, which consists of epithelial cells, provides selective permeability, which allows only essential nutrients and prevents the entry of harmful components, such as 
bacteria and its toxins, from the intestinal lumen (Lee et al., 2015). Any damage to intestinal cells breaches the barrier, and results in the entry of harmful substances that may lead to the shortening of villi and epithelial sloughing (Sikandar et al., 2017). The absorption of nutrients across the intestine is increased greatly by the intestinal villi and microvilli (Awad et al., 2009). Intestinal health and integrity are associated with villus height, width, surface area, and villus height to crypt depth ratio, which are the important indicators of intestinal digestion and absorption ( $\mathrm{Li}$ et al., 2018). The literature about the effects of chromium on intestinal histology in broilers is scarce. In the current research, the villus height of duodenum and jejunum was found to be significantly higher in birds supplemented with $800 \mu \mathrm{g} \mathrm{Cr}$-CNPs but remained unaffected in the ileum. Crypt depth of duodenum in birds supplemented with 200 and $400 \mu \mathrm{g} \mathrm{Cr}$-CNPs $/ \mathrm{kg}$ was found to be higher $(P<0.001)$ compared with other supplemented and control groups. The jejunal and ileal crypt depth remained unaffected with supplementation compared with the control group. The duodenal and ileal villus surface area remained unaffected with Cr-CNP supplementation. The jejunal villus surface area of birds supplemented with 400 and $800 \mu \mathrm{g} C r-C N P s / k g$ was found higher $(P<0.001)$ compared with the control group. The villus height to crypt depth ratio of duodenum and ileum remained unaffected with supplementation compared with the control group. Jejunal villus height to crypt depth ratio of birds supplemented with $800 \mu \mathrm{g} \mathrm{Cr}$-CNPs/kg was found to be higher $(P<0.001)$ compared with the $1200 \mu \mathrm{g} \mathrm{Cr}$ CNPs/kg supplemented group. Li et al. (2018) conducted a study to observe the effects of Cr-picolinate in duck reared under heat stress and found that Cr-picolinate did not affect the villus height and crypt depth in duodenum, jejunum, and ileum at days 14, 21 and 35. However, the villus height to crypt depth ratio was increased significantly with $\mathrm{Cr}$-picolinate supplementation in the jejunum and ileum.

\section{Conclusion}

Supplemental Cr-CNPs did not affect growth and development of the viscera or cause hepatic damage or nephrotoxicity. Supplementation with Cr-CNPs did affect retention of chromium in the bone. Intestinal histology was also improved with Cr-CNP supplementation. However, future investigations are needed to gain insight into the exact mechanism that underlies the effects of supplemental Cr-CNPs in broilers.

\section{Acknowledgements}

This research was funded by Pakistan Agricultural Research Council-Islamabad, under Agricultural Linkages Program (ALP Project No. AS-091).

\section{Authors' Contributions}

SKT, MSY, MAR and HR participated in the research design and conduction of an experimental trial. AFK helped in the preparation and characterization of nanoparticles. SKT, SA, HZ and IK participated in the data collection and laboratory analyses. SKT, MSY and HR analysed the data and prepared the manuscript.

\section{Conflict of Interest Declaration}

The authors declare that they have no conflict of interest.

\section{References}

Akbari, M. \& Torki, M., 2014. Effects of dietary chromium picolinate and peppermint essential oil on growth performance and blood biochemical parameters of broiler chicks reared under heat stress conditions. Int. J. Biometeorol. 58,1383-1391. DOI 10.1007/s00484-013-0740-1

Amatya, J., Haldar, S. \& Ghosh, T., 2004. Effects of chromium supplementation from inorganic and organic sources on nutrient utilization, mineral metabolism and meat quality in broiler chickens exposed to natural heat stress. J. Anim. Sci. 79(2), 241-253. DOI: https://doi.org/10.1017/S135772980009010X

Ashraf, S., Zaneb, H., Yousaf, M., ljaz, A., Sohail, M., Muti, S., Usman, M., ljaz, S. \& Rehman, H., 2013. Effect of dietary supplementation of prebiotics and probiotics on intestinal microarchitecture in broilers reared under cyclic heat stress. J. Anim. Physiol. Anim. Nutr. 97(1), 68-73. https://doi.org/10.1111/jpn.12041

Awad, R., Abou-Aly, A., Gawad, M.A. \& G-Eldeen, I., 2012. The influence of SnO2 nano-particles addition on the Vickers microhardness of (Bi, Pb)-2223 superconducting phase. J. Supercond. Nov. Magn. 25(4), 739-745. DOI 10.1007/s10948-011-1334-y

Awad, W.A., Ghareeb, K., Abdel-Raheem, S. \& Bohm, J., 2009. Effects of dietary inclusion of probiotic and symbiotic on growth performance, organ weights, and intestinal histomorphology of broiler chickens. Poult. Sci. 88, 49-55. https://doi.org/10.3382/ps.2008-00244

Bakhiet, A.O. \& Elbadwi, S.M.A., 2007. Effects of dietary chromium supplementation on the performance and some serum parameters in Bovans-type chicks. J. Pharmacol. Toxicol. 2, 402-406. https://www.researchgate.net/profile/Amel_Bakhiet/publication/268354690

Chang, X., Mowat, D.N. \& Spiers. G.A., 1992. Carcass characteristics and tissue-mineral contents of steers fed supplemental chromium. Can. J. Anim. Sci. 72, 663-668. https://doi.org/10.4141/cjas92-077

Desai, M.P., Labhasetwar, V., Amidon, G.L. \& Levy, R.J., 1996. Gastrointestinal uptake of biodegradable microparticles: effect of size. Pharma. Res. 13(12), 1838-1845. DOI: 10.1023/a:1016085108889 
Ghanbari, S., Ebrahimnazhad, Y., Eshratkah, B. \& Nazeradl, K., 2012. Effect of dietary chromium supplementation on performance and carcass traits of broiler chicks. Pak. J. Nutr. 11, 467-472. http://docsdrive.com/pdfs/ansinet/pjn/2012/467-472.pdf

Giambron, J.J. \& Clay, R.P., 1986.Vaccination of day-old broiler chicks against Newcastle disease and infection bursal disease using live and/or inactivated vaccines. Avian Dis. 30, 557-561. DOI: 10.2307/1590421

Habibian, M., Ghazi, S. \& Moeini, M.M., 2013. Lack of effect of dietary chromium supplementation on growth performance and serum insulin, glucose, and lipoprotein levels in broilers reared under heat stress condition. Biol. Trace. Elem. Res. 153(3), 205-211. DOI 10.1007/s12011-013-9663-2

Hamidi, O., Chamani, M., Ghahri, H., Sadeghi, A.A. \& Mealekinejad, H., 2016. Effects of chromium(III) picolinate and chromium(III) picolinate nanoparticles supplementation on growth performance, organs weight and immune function in cyclic heat stressed broiler chickens. Kafkas Univ. Vet. Fak. Derg. 22, 373-380. DOI: $10.9775 / \mathrm{kvfd} .2015 .14736$.

He, Q., Mitchell, A.R., Johnson, S.L., Wagner-Bartak, C., Morcol, T. \& Bell, S.J., 2000. Calcium phosphate nanoparticle adjuvant. Clin. Diagnos. Lab. Immunol. 7(6), 899-903. DOI: 10.1128/CDLI.7.6.899-903.2000

Hirano, S., Itakura, C., Seino, H., Akiyama, Y., Nonaka, I., Kanbara, N. \& Kawakami, T., 1990. Chitosan as an ingredient for domestic animal feeds. J. Agric. Food Chem. 38(5), 1214-1217. https://doi.org/10.1021/jf00095a012

Hussain, N., Jaitley, V. \& Florence, A.T., 2001. Recent advances in the understanding of uptake of microparticulates across the gastrointestinal lymphatics. Adv. Drug Del. Rev. 50(1), 107-142. PII: S0169-409X(01)00152-1

Kegley, E.B., Spears, J.W. \& Brown, T.T., 1996. Immune response and disease resistance of calves fed chromium nicotinic acid complex or chromium chloride. J. Dairy Sci. 79, 1278-1283. https://doi.org/10.3168/jds.S00220302(96)76482-2

Khambualai, O., Yamauchi, K., Tangtaweewipat, S. \& Cheva-Isarakul, B., 2009. Growth performance and intestinal histology in broiler chickens fed with dietary chitosan. Br. Poult. Sci. 50(5), 592-597. https://doi.org/10.1080/ 00071660903247182

Khan, I., Zaneb, H., Masood, S., Yousaf, M.S., Rehman, H.F. \& Rehman, H., 2017. Effect of Moringa oleifera leaf powder supplementation on growth performance and intestinal morphology in broiler chickens. J. Anim. Physiol. Anim. Nutr. 101, 114-121. https://doi.org/10.1111/jpn.12634

Kroliczewska, B., Zawadzki, W., Dobrzanski, Z. \& Kaczmarek-Oliwa, A., 2004. Changes in selected serum parameters of broiler chicken fed supplemental chromium. J. Anim. Physiol. Anim. Nutr. 83, 393-400. https://doi.org/10.1111/j.1439-0396.2004.00496.x

Lee, K.W., Kim, D.K., Lillehoj, H.S., Jang, S.I. \& Lee, S.H., 2015. Immune modulation by Bacillus subtilis-based direct-fed microbials in commercial broiler chickens. Anim. Feed. Sci. Technol. 200, 76-85. https://doi.org/10.1016/j.anifeedsci.2014.12.006

Li, R., Zhou, Y., Li, Y., Guo, L., Zhang, Y. \& Qi, Z., 2018. Effects of chromium picolinate supplementation on growth performance, small intestine morphology and antioxidant status in ducks under heat stress conditions. Int. J. Morphol. 36(1), 226-234. http://www.intjmorphol.com/wp-content/uploads/2018/02/art_38_361.pdf

Lien, T., Horng, Y. \& Yang, K., 1999. Performance, serum characteristics, carcase traits and lipid metabolism of broilers as affected by supplement of chromium picolinate. br. poult. sci. 40(3), 357-363. https://doi.org/10.1080/00071669987458

Lin, Y., Huang, J., Li, M., Cheng, C. \& Lien, T., 2015. Effects of supplemental nanoparticle trivalent chromium on the nutrient utilization, growth performance and serum traits of broilers. J. Anim. Physiol. Anim. Nutr. 99(1), 59-65. https://doi.org/10.1111/jpn.12215

Lindemann, M., Wood, C., Harper, A., Kornegay, E. \& Anderson, R., 1995. Dietary chromium picolinate additions improve gain: feed and carcass characteristics in growing-finishing pigs and increase litter size in reproducing sows. J. Anim. Sci. 73(2), 457-465. https://doi.org/10.2527/1995.732457x

Mertz, W., 1993. Chromium in human nutrition: A review. J. Nutr. 123(4), 626-633. https://doi.org/10.1093/jn/123.4.626.

Moeini, M.M., Bahrami, A., Ghazi, S. \& Targhibi, M.R., 2011. The effects of different levels of organic and inorganic chromium supplementation on production performance, carcass traits and some blood parameters of broiler chicken under heat stress condition. Biol. Trace. Elem. Res. 144, 715-724. DOI 10.1007/s12011-011-9116-8

Mooney, K. \& Cromwell, G., 1995. Effects of dietary chromium picolinate supplementation on growth, carcass characteristics, and accretion rates of carcass tissues in growing-finishing swine. J. Anim. Sci. 73(11), 3351-3357. https://doi.org/10.2527/1995.73113351x

Motozono, Y., Hatano, K., Sugawara, N. \& Ishibashi, T., 1998. Effects of dietary chromium picolinate on growth, carcass quality and serum lipids of female broilers. Anim. Sci. Technol. 69, 659-665. http://agris.fao.org/agrissearch/search.do?recordID=JP1998007126

Page, T., Southern, L., Ward, T. \& Thompson, D., 1993. Effects of chromium picolinate on growth and serum and carcass traits of growing-finishing pigs. J. Anim. Sci. 71 (3): 656-662. https://doi.org/10.2527/1993.713656x.

Rajalekshmi, M., Sugumar, C., Chirakkal, H. \& Ramarao, S.V., 2014. Influence of chromium propionate on the carcass characteristics and immune response of commercial broiler birds under normal rearing conditions. Poult. Sci. 93, 574-580. https://doi.org/10.3382/ps.2013-03373

Rosebrough, R.W. \& Steele, N.C., 1981. Effect of supplemental dietary chromium or nicotic acid on carbonhydrate metabolism during basal, starvation and refeeding periods in poults. Poult. Sci. 60, 407-411. https://doi.org/10.3382/ps.0600407.

Sahin, K., Sahin, N. \& Kucuk, O., 2002. Effects of dietary chromium and ascorbic acid supplementation on digestion of nutrients, serum antioxidant status, and mineral concentrations in laying hens reared at a low ambient temperature. Biol. Trace Elem. Res. 87(3), 113-124. DOI: 10.1385/BTER:87:1-3:113 
Sahin, N., Sahin, K., Onderci, M., Gursu, M., Cikim, G., Vijaya, J. \& Kucuk, O., 2005. Chromium picolinate, rather than biotin, alleviates performance and metabolic parameters in heat-stressed quail. Br. Poult. Sci. 46(4), 457-463. DOI: 10.1080/00071660500190918

Samanta, S., Haldar, S. \& Ghosh, T.K., 2008. Production and carcase traits in broiler chickens given diets supplemented with inorganic trivalent chromium and an organic acid blend. Br. Poult. Sci. 49, 155-163. DOI: 10.1080/00071660801946950

Shi, B., Li, D., Piao, X. \& Yan, S., 2005. Effects of chitosan on growth performance and energy and protein utilisation in broiler chickens. Br. Poult. Sci. 46(4), 516-519. https://doi.org/10.1080/00071660500190785

Shi-bin, Y. \& Hong, C., 2012. Effects of dietary supplementation of chitosan on growth performance and immune index in ducks. Afr. J. Biotechnol. 11(14), 3490-3495.

Sikandar, A., Zaneb, H., Younus, M., Khattak, F., Masood, S., Aslam, A., Ashraf, S., Rehman, H. \& Yousaf, M.S., 2017. Effect of sodium butyrate on performance, immune status, microarchitecture of small intestinal mucosa and lymphoid organs in broiler chickens. Asian-Australas. J. Anim. Sci. 30(5), 690-699. DOI: 10.5713/ajas.16.0824

Sirirat, N., Lu, J.J., Hung, A.T.Y., Chen, S.Y. \& Lien, T.F., 2012. Effects of different levels of nanoparticles chromium picolinate supplementation on growth performance, mineral retention, and immune responses in broiler chickens. J. Agric. Sci. 4(12), 48-58. DOI:10.5539/jas.v4n12p48

Toghyani, M., Toghyani, M., Shivazad, M., Gheisari, A. \& Bahadoran, R., 2012. Chromium supplementation can alleviate the negative effects of heat stress on growth performance, carcass traits, and meat lipid oxidation of broiler chicks without any adverse impacts on blood constituents. Biol. Trace Elem. Res. 146(2), 171-180. DOI: 10.1007/s12011-011-9234-3

Uyanik, F., Atasever, A., Özdamar, S. \& Aydin, F., 2002. Effects of dietary chromium chloride supplementation on performance, some serum parameters, and immune response in broilers. Biol. Trace Elem. Res. 90(1-3), 99-115. https://link.springer.com/content/pdf/10.1385\%2FBTER\%3A90\%3A1-3\%3A99.pdf

Wang, M. \& Xu, Z., 2004. Effect of chromium nanoparticle on growth performance, carcass characteristics, pork quality and tissue chromium in finishing pigs. Asian-Austral. J. Anim. Sci. 17 (8), 1118-1122. https://doi.org/10.5713/ajas.2004.1118

Wang, M., Xu, Z., Zha, L. \& Lindemann, M., 2007. Effects of chromium nanocomposite supplementation on blood metabolites, endocrine parameters and immune traits in finishing pigs. Anim. Feed Sci. Technol. 139 (1), 69-80. https://doi.org/10.1016/j.anifeedsci.2006.12.004

Wang, M-Q., Wang, C., Li, H., Du, Y.J., Tao, W.J., Ye, S.S. \& He, Y.D., 2012. Effects of chromium-loaded chitosan nanoparticles on growth, blood metabolites, immune traits and tissue chromium in finishing pigs. Biol. Trace Elem. Res. 149, 197-203. DOI 10.1007/s12011-012-9428-3

Yousaf, M.S., Rahman Z.U., Sandhu M.A., Bukhari, S.A. \& Yousaf A., 2009. Comparison of the fast-induced and high dietary zinc-induced molting: trace elements dynamic in serum and eggs at different production stages in hens (Gallus domesticus). J. Anim. Physiol. Anim. Nutr. 93 (1), 35-43. DOI: 10.1111/j.1439-0396.2007.00775.x

Zha, L.Y., Zeng, J.W., Chu, X.W., Mao, L.M. \& Luo, H.J., 2009. Efficacy of trivalent chromium on growth performance, carcass characteristics and tissue chromium in heat-stressed broiler chicks. J. Sci. Food Agric. 89(10), 17821786. https://doi.org/10.1002/jsfa.3656

Zheng, C., Huang, Y., Xiao, F., Lin, X. \& Lloyd, K., 2016. Effects of supplemental chromium source and concentration on growth, carcass characteristics, and serum lipid parameters of broilers reared under normal conditions. Biol. Trace Elem. Res. 169, 352-358. DOI: 10.1007/s12011-015-0419-z 